University of Wollongong

Research Online

Faculty of Engineering and Information

Faculty of Engineering and Information

Sciences - Papers: Part A

Sciences

$1-1-2015$

\title{
Transparent and conformal 'piezoionic' touch sensor
}

Mirza Sarwar

University of British Columbia

Yuta Dobashi

University of British Columbia

Ettore Glitz

University of British Columbia

Meisam Farajollahi

University of British Columbia

Shahriar Mirabbasi

University of British Columbia

See next page for additional authors

Follow this and additional works at: https://ro.uow.edu.au/eispapers

Part of the Engineering Commons, and the Science and Technology Studies Commons

Research Online is the open access institutional repository for the University of Wollongong. For further information contact the UOW Library: research-pubs@uow.edu.au 


\title{
Transparent and conformal 'piezoionic' touch sensor
}

\begin{abstract}
A polyurethane hydrogel based touch sensor with high transparency and conformability is demonstrated. Polyurethane hydrogels swollen with various electrolytes were compressed at a pressure of $30 \mathrm{kPa}$, simulating a fingertap on a conventional touch screen device. Unlike ionic polymer metal composite and conducting polymer trilayer sensors, where electrodes render the sensors opaque and relatively rigid, the electrodes used in this work are metal wires or strips, separated from each other by regions of transparent film, enabling transparency and compliance. The voltages and currents observed when the perturbation is above one electrode are on the order of $10(-2) \mathrm{V}$ and $10(-7) \mathrm{A}$, relative to a second electrode that is approximately $1 \mathrm{~cm}$ away. The sign of voltage and current signals detected from perturbations made between electrodes is determined by relative proximity to each electrode, and the magnitude appears to decrease with increasing distance from the electrodes. These observations suggest that it may be possible to discriminate the location of touch based on signals transmitted to the edges of an ionically conductive film. A model to describe the inhomogeneous ionic distribution and predict the resultant voltage and current is presented to qualitatively explain the sensing, based on the Donnan potential.
\end{abstract}

\section{Keywords}

touch, piezoionic, sensor, conformal, transparent

Disciplines

Engineering | Science and Technology Studies

\section{Publication Details}

Sarwar, M. Saquib us., Dobashi, Y., Glitz, E. F. Scabeni., Farajollahi, M., Mirabbasi, S., Nafici, S., Spinks, G. M. \& Madden, J. D.W. (2015). Transparent and conformal 'piezoionic' touch sensor. In Y. Bar-Cohen (Eds.), Proceedings of SPIE: Electroactive Polymer Actuators and Devices (EAPAD) 2015 (pp. 943026-1-943026-9). United States: S P I E - International Society for Optical Engineering.

\section{Authors}

Mirza Sarwar, Yuta Dobashi, Ettore Glitz, Meisam Farajollahi, Shahriar Mirabbasi, Sina Nafici, Geoffrey M. Spinks, and John D. Madden 


\title{
Transparent and Conformal 'PiezoIonic' Touch Sensor
}

\author{
Mirza Saquib us Sarwar ${ }^{1}$, Yuta Dobashi ${ }^{1}$, Ettore F. Scabeni Glitz ${ }^{1}$, Meisam Farajollahi ${ }^{2}$, Shahriar \\ Mirabbasi ${ }^{1}$, Sina Nafici ${ }^{3}$, Geoffrey M. Spinks ${ }^{3}$, John D. W. Madden* \\ 1 Department of Electrical and Computer Engineering, University of British Columbia, \\ Vancouver, BC V6T 1Z4 \\ 2 Department of Mechanical Engineering, University of British Columbia, \\ Vancouver, BC V6T 1Z4 \\ ${ }^{3}$ ARC Center of Excellence in Electromaterials Science, University of Wollongong, Wollongong, \\ Australia. \\ * Advanced Materials and Process Engineering Laboratory, Department of Electrical and Computer \\ Engineering, University of British Columbia, Vancouver, BC V6T1Z4 \\ Email: jmadden@ece.ubc.ca, Tel: +1-604-827-5306
}

\begin{abstract}
A polyurethane hydrogel based touch sensor with high transparency and conformability is demonstrated. Polyurethane hydrogels swollen with various electrolytes were compressed at a pressure of $30 \mathrm{kPa}$, simulating a fingertap on a conventional touch screen device. Unlike ionic polymer metal composite and conducting polymer trilayer sensors, where electrodes render the sensors opaque and relatively rigid, the electrodes used in this work are metal wires or strips, separated from each other by regions of transparent film, enabling transparency and compliance. The voltages and currents observed when the perturbation is above one electrode are on the order of $10^{-2} \mathrm{~V}$ and $10^{-7} \mathrm{~A}$, relative to a second electrode that is approximately $1 \mathrm{~cm}$ away. The sign of voltage and current signals detected from perturbations made between electrodes is determined by relative proximity to each electrode, and the magnitude appears to decrease with increasing distance from the electrodes. These observations suggest that it may be possible to discriminate the location of touch based on signals transmitted to the edges of an ionically conductive film. A model to describe the inhomogeneous ionic distribution and predict the resultant voltage and current is presented to qualitatively explain the sensing, based on the Donnan potential.
\end{abstract}

Keywords: flexible, stretchable, electronics, transparent, touch sensor,

\section{INTRODUCTION}

Transducers capable of detecting and/or measuring pressure are ubiquitous. Conventional sensing technologies include piezoresistive [1]-[3], piezoelectric [4], [5], capacitive [6]-[8], optical [9], and magnetic [10] methods. The wellestablished sensors are not generally highly stretchable and transparent - properties that are increasingly sought for wearable applications among others.

Increasingly sensors are being incorporated onto thermoplastic (bendable) and elastomeric (stretchable) substrates. Promising capacitance based technology is shown to be highly sensitive [11], stretchable by up to $150 \%$ [12] [13], and relatively transparent [12] [13]. However these promising approaches still have some limitations for widespread use as they either employ poorly stretchable electrodes [11], or emerging materials such as CNT and AgNW that are not yet mass produced. Sun et al [14] employ electrolyte containing tough gels to show very highly stretchable (2000\%) and transparent (98.9\%) capacitive sensors. It is a capacitive sensor formed by a trilayer of a layer of transparent elastomer forming the dielectric, between ionically conductive transparent polymer layers which act as stretchable electrodes. These, in addition to other sensor technologies being developed currently face challenges such as cost and power requirement or complexity of readout circuit, and have to settle for trade-offs between transparency, flexibility, complexity and cost. We demonstrate a sensor that is highly deformable and requires only mechanical energy input to produce a signal, hence no energy spent in generating a sense signal.

A touch sensor is introduced that employs tough, compliant, and transparent polyurethane hydrogels. Cross-linked hydrogel materials have been used in many applications such as separation membranes, biosensors, valves, artificial muscle [15], [16], drug delivery devices [17], super-adsorbent nano-composites [18] and actuators for optics and fluidics

Electroactive Polymer Actuators and Devices (EAPAD) 2015, edited by Yoseph Bar-Cohen, Proc. of SPIE Vol. 9430, $943026 \cdot$ C 2015 SPIE · CCC code: 0277-786X/15/\$18 · doi: 10.1117/12.2085598 
[19]. Hydrogels are very soft and wet material with low sliding friction [20] and high solute permeability [21]. The photographs in Figure 1 show a typical sample used in this work. The gel is compliant Figure 1 (b), conformal to surfaces (c and d), and over $90 \%$ transparent (e). The elastic modulus is approximately $300 \mathrm{kPa}$.

The gels that are employed in this work contain mobile ions that are displaced in response to applied force. The physical mechanisms may be similar to those in bending and stretching sensors based on conducting polymers and Ionic Polymer Metal Composites (IPMCs). Takashima et al [22] referred to the sensor response as the "Mechano-chemo-electrical Effect". His work showed that current is generated by a polyaniline film when a step in force is applied. Peak currents of $5 \mu \mathrm{A}$ were observed in response to loads of several $\mathrm{MPa}$, and charge transferred was $7 \mu \mathrm{C}$ in a $30 \mu \mathrm{m}$ thick, $10 \mathrm{~mm}$ long and $2 \mathrm{~mm}$ wide film. Charge increased linearly with the applied stress at approximately $2.5 \mathrm{mC} / \mathrm{m}^{3}$ per Pascal. More recent work with conducting polymers has shown this effect in bending trilayers [23], [24]. This work suggested that deformation of the material leads to ion concentration gradients that in turn generate so-called Donnan potentials. Alternatively the pressure gradient induced in the material may lead directly to a displacement of ions [25].

Shahinpour demonstrates a bending sensor based on ionic polymer gels [26], with electrodes covering the top and bottom of the gel film. A voltage is generated when this trilayer film is deflected. Ionic polymer metal composites form similar structures in which the electrodes on the top and bottom are now highly porous, and surround a polyelectrolyte such as NafionTM. These are also known to develop voltages and currents when they are bent [27], with the composite generating voltage in response to bending induced differential stress within the thickness of the film. In these materials only mechanical energy input is needed to generate voltage or current, in analogy to the effect in piezoelectric materials. For this reason, we propose that this class of ionic sensors be referred to as Piezoionic. Like piezoelectric materials, they can also be actuated by external voltage. In analogy to piezoelectrics, there is a coefficient relating applied stress to voltage, as well as applied charge to strain [25], with a key difference being that ions (free charges) are mobile, unlike the dielectric charges in piezoelectrics.

In the subsequent section of the paper a mechanism is proposed to explain the piezoionic effect. The magnitude of voltage generated can be predicted based on similar mechanisms to those responsible for the Donnan potential across a biological cell membrane. In this case the mechanism is thought to result from changes in local concentration as the material is locally compressed. Experimental work shows the conceptual alignment with the postulate. It also demonstrates the capability to distinguish spatial positioning of touch.

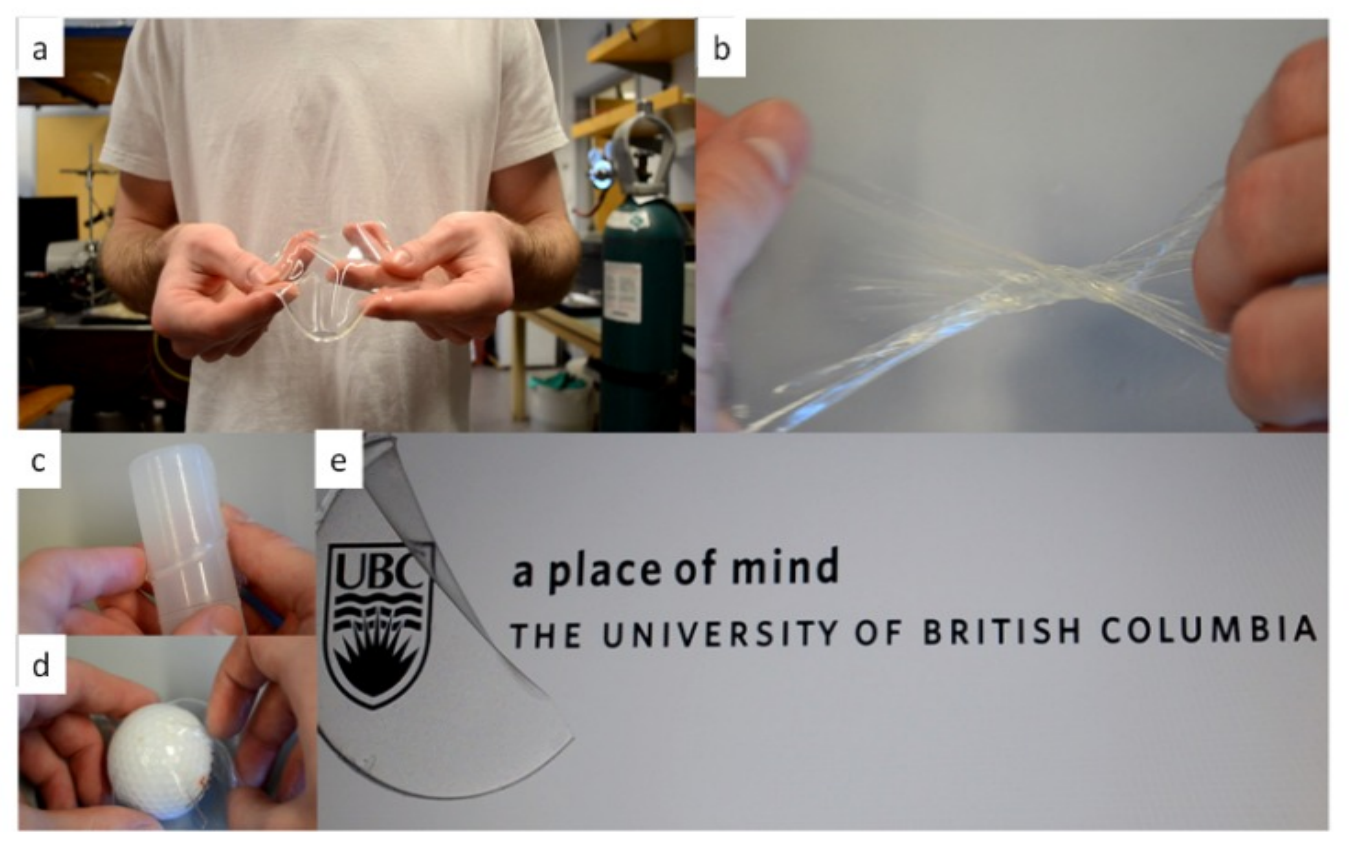

Figure 1: Piezoionic material showing conformity, transparency and mechanical strength 


\section{PROPOSED MECHANISM OF PIEZOIONIC EFFECT}

The piezoionic effect may be a result of Donnan like depolarization due to an inhomogeneous ionic distribution. The highly deformable polymeric ion host initially at equilibrium has a uniform distribution of mobile species such that the electrochemical potential experienced by all species is equal and the free energy of the system is minimal.

When a mechanical perturbation causes the polymer matrix to non-homogeneously deform as shown in Figure 2, the ionic species will experience a differential pressure locally, and displace such that the chemical potential change compensates the pressure applied which is described by Gibbs-Duhem equation. At this depolarized state, the change in chemical potential must directly correspond to the change in electrical potential measured. This electrical potential change, $\Delta \mathrm{E}$, can be measured using electrodes that are in contact with the material.

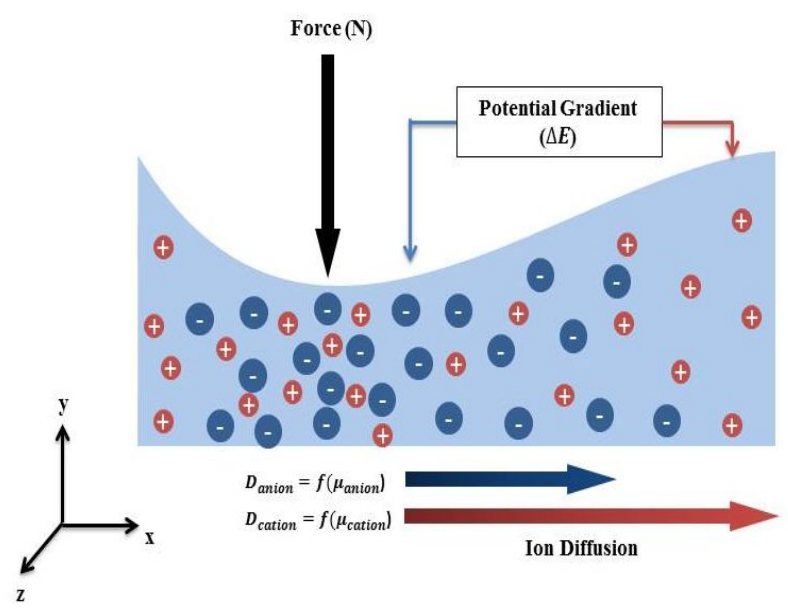

Figure 2: Piezoionic effect showing inhomogeneous ionic distribution

To predict the voltage induced by the deformation the electromechanical coupling must be explained by means of available free energy. We begin with the Gibbs-Duhem equation in a poroelastic solid:

$$
-\varphi V d P+s d T+\sum_{i} n_{i} d \mu_{i}=0
$$

$\varphi$ is the porosity of the polymer, $\mathrm{dP}$ is the infinitesimal pressure change within the bulk, $\mathrm{s}$ is the entropy of the overall system, $T$ is the temperature, $n_{-} i$ is the number of moles of species $i$, and finally $d \mu \_i$ represents the infinitesimal change in the chemical potential of species i. Here we assume a constant temperature system, eliminating the entropic effect:

$$
\varphi V d P=\sum_{i} n_{i} d \mu_{i}
$$

The term on the right hand side is simply the partial molar Gibbs free energy, and we may now equate the mechanical pressure change applied to the polymer bulk to ion concentration distribution assuming a non-interacting system:

$$
d G=\varphi V d P=R T \ln \frac{[i]_{x}}{[i]_{x+\Delta x}}
$$

Where [i] represents the concentration of the ith species and the subscripts represent two locations separated by a distance $\Delta \mathrm{x}$ within the sample. To associate the ion concentration to the resultant voltage, we take the approach of solving for Donnan potential induced by inhomogeneous ion distribution across a cellular membrane in biological systems. The electrochemical potential of species i can be expressed as a summation of chemical and Volta potentials as follows:

$$
\bar{\mu}=\mu+z F E=R T \ln \left[i^{+}\right]+z F E
$$


$\mathrm{z}$ is the valence charge of the ion, $\mathrm{F}$ is Faraday's constant, and $\mathrm{E}$ is the electrical potential. When the polymer is deformed and the mobile species attains a new equilibrium, the electrochemical potential is constant across the system. Thus the chemical potential change induced by pressure gradient must be directly compensated by the change in electrical potential:

$$
\begin{gathered}
\Delta \bar{\mu}=\Delta \mu+z F \Delta E=0 \\
\Delta E=\frac{R T}{z F} \ln \left(\frac{\left[i^{+}\right]_{x}}{\left[i^{+}\right]_{x+\Delta x}}\right)
\end{gathered}
$$

The equation predicts that, for example, if concentration of a species varies by a factor of 2.718 , the potential difference produced will $25 \mathrm{mV}$ at room temperature. This could occur for example if the gel is highly compressed, producing a local increase in concentration.

The voltage resulting from a steady mechanical deformation can persist providing either cations or anions are immobilized onto the polymer backbones such as seen in IPMC based configurations [28]. If both cationic and anionic species are mobile, signal may arise due to differences in diffusion coefficients and mobilities between species. This response might be described by the Goldman-Hodgkin-Katz equation, which describes the Donnan potential in terms of the ion concentrations as well as their permeabilities, $\mathrm{P}_{\mathrm{i}}$, across a biological membrane:

$$
\Delta E=\frac{R T}{z F} \ln \left(\frac{\sum_{i}^{N} P_{\text {cation }_{i}}\left[\text { cation }_{i}\right]_{x}+\sum_{i}^{M} P_{\text {anion }_{i}}\left[\text { anion }_{i}\right]_{x+\Delta x}}{\left.\sum_{i}^{N} P_{\text {cation }_{i}}\left[\text { cation }_{i}\right]_{x+\Delta x}+\sum_{i}^{M} P_{\text {anion }_{i}} \text { anion }_{i}\right]_{x}}\right) .
$$

The potential drops to zero, or the original open circuit potential of the cell, over time if both anions and cations are mobile. In the gel sensors used in this work one mobile anion and one mobile cation are present. A generation of a signal of a particular polarity is interpreted as indicating a higher permeability of the matrix to one sign or ion compared to the other.

\section{CHARACTERIZATION}

The magnitude of electrical signal generated is now demonstrated using polyurethane gels with two mobile ions, two electrodes in contact with the gel and a compressive perturbation applied normal to the surface of the gel.

\section{Fabrication of the hydrogel film samples}

Water soluble urethane HydroMedTMD640 (AdvanSource Polymer, USA) is used. $2 \mathrm{~g}$ of the polymer is mixed with 1 $\mathrm{ml}$ of deionized water and $17 \mathrm{ml}$ of ethanol. A highly viscous precursor is formed after stirring constantly over a period of 24 hours. A glass Petri dish is used as the mold, and it is sealed with perforated Parafilm ${ }^{\circledR}$ to enable slow evaporation of ethanol at room temperature. The sample dries completely after 48 hours, and is readily removed from the mold. Subsequently, the dry hydrogel film is immersed in deionised water for $20-30$ minutes. The hydrophilic carboxyl and hydroxyl functional groups facilitate the water absorption, resulting in up to $300 \mathrm{vol} \%$ increase. The swollen hydrogel films are placed in aqueous solutions of sodium chloride, potassium chloride, and sodium hexafluorophosphate for at least 24 hours.

Young's Modulus of the hydrogel was measured to be $0.3 \mathrm{MPa}$ at $0.1 \mathrm{~Hz}$ sine wave strain of up to $10 \%$. The hydrogel undergoes a maximum of $100 \%$ linear expansion. It contains up to $90 \mathrm{wt} \%$ water at $200-300 \mathrm{vol} \%$ swelling ratio. High strain and swelling ratio make this material suitable to use as reservoir of ions and a flexible substrate.

\section{Response characterization}

To characterize the generation properties of the hydrogel sensor a Bose ElectroForce ${ }^{\circledR} 3100$ tension/compression test instrument is used to apply controlled pressure on the sample. The hydrogel film is placed on a microscopic glass slide with two stainless steel wire electrodes $(\mathrm{d}=250 \mu \mathrm{m})$ placed $1 \mathrm{~cm}$ apart underneath. The electrodes are connected to an AutoLab ${ }^{\circledR}$ Potentiostat/Galvanostat as shown in Figure 4 (a). The hydrogel was pressed on by a stiff cylindrical rod $(\mathrm{d}=$ $5 \mathrm{~mm}$ ) firmly attached to the actuator. The film was perturbed for $200 \mathrm{~ms}$ with pressure of $30 \mathrm{kPa}$. A typical force cycle is illustrated in Figure 4 (b).

The force applied as well as the resultant displacement were recorded by the Wintest ${ }^{\circledR}$ software, which was further processed in MATLAB ${ }^{\circledR}$ to calculate the strain induced at every perturbation. AutoLab ${ }^{\circledR}$ was set in potentiostat mode to record the short circuit current (sampling rate: $100 \mathrm{~Hz}$ ), and gavlanostat mode to record the open circuit potential (sampling rate: $4 \mathrm{~Hz}$ ) throughout the perturbation sequence. 

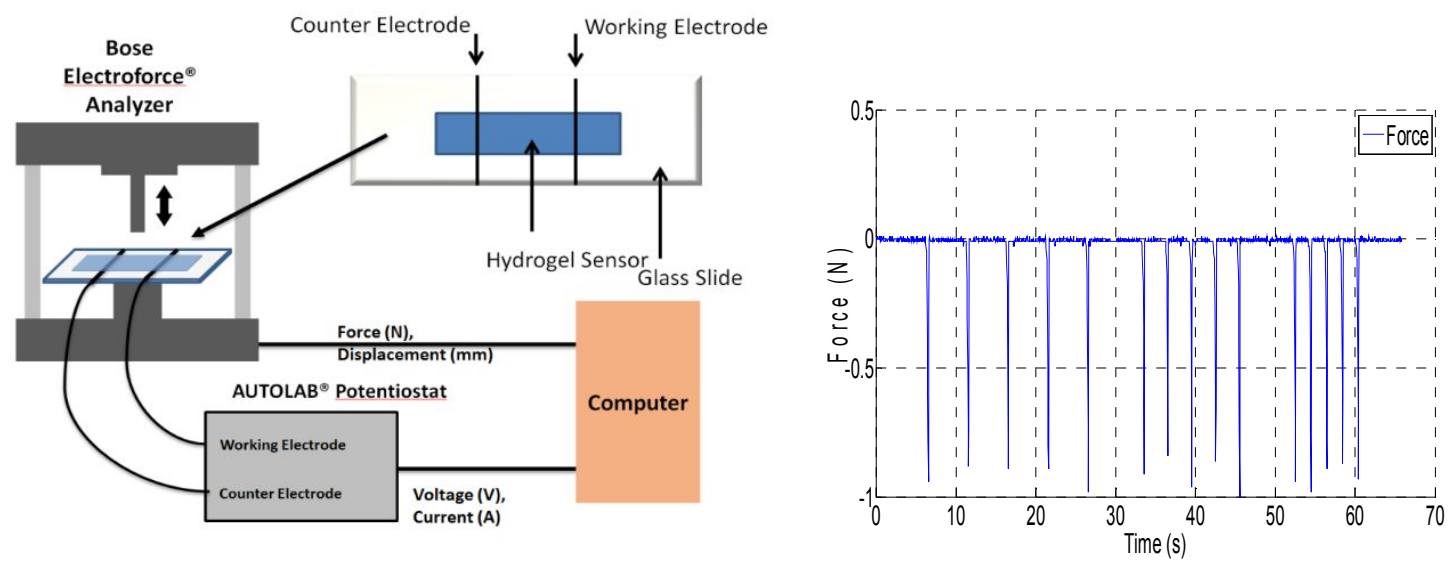

Figure 3: (a) System setup (b) Sample sequence of taps used in characterization of hydrogel sensor

\section{RESULTS}

Figure 4 below illustrates the voltage signal generated by this sequence of 15 perturbations on a hydrogel film of dimensions $8 \mathrm{~mm} \times 3 \mathrm{~cm} \times 200 \mu \mathrm{m}$, and contains $1.0 \mathrm{M} \mathrm{KCl}$ aqueous salt. Electrodes were stainless steel wires placed 1 $\mathrm{cm}$ apart. The open circuit voltage at rest state is presented as observed. The instantaneous response to perturbations, as seen by the sudden peaks in open circuit voltage, is as fast as the mechanical perturbation $(\sim 20 \mathrm{~ms})$. The potential subsequently returns towards its initial value over several seconds. This relaxation is likely due to i) the differential diffusion coefficients of the mobile species and or ii) the mechanical relaxation time of the gel. The polarity of the change depends of location of the perturbations relative to each electrode. At the left hand electrode (counter electrode) voltage drops upon application of pressure, presumably due to the preferential expulsion of positive ions (in this case $\mathrm{K}^{+}$), as depicted in Figure 4 (a). The opposite is seen when perturbations are performed on the right hand electrode (working electrode). The magnitudes of peaks in voltage are as large as $13 \mathrm{mV}$. Perhaps due to the limits in the mechanical relaxation time of the sample, subsequent perturbations are attenuated compared to the beginning of the sequence. There is some recovery of the voltage signal after a pause in perturbation, e.g. the one that takes place around $55 \mathrm{~s}$, while higher frequency perturbations appears to be associated with reduced signal. When perturbations are made close to the middle of the sensor, and away from electrodes, the signal is smaller (note smaller scale). In principle this signal would be zero if the perturbation is made exactly in the middle, but despite many attempts we have been unable to produce complete cancellation.

The short circuit current response for the same perturbation sequence is shown in Figure 5. Response time is again fast $(20 \mathrm{~ms})$. Once again polarity is reversed when taps are induced on the opposing electrode. The polarity of voltages (Figure 4) and currents (Figure 5) suggest that $\mathrm{K}^{+}$ions are more mobile in this situation than $\mathrm{Cl}^{-}$. The current observed at the electrodes shows both the effect of the initial perturbation, and the reversal of the current as the perturbation ends. The recovery of the current to zero is faster compared to the voltage relaxation time after a perturbation. The current signal generated between electrodes is smaller than at the electrodes (note the smaller scale).

What is the effect of the position of the tap relative to the electrodes? Results are shown below in Figure 6. The electrodes in this case were $8 \mathrm{~mm}$ wide copper strips and were $50 \mathrm{~mm}$ apart. A film of hydrogel with a thickness of 200 $\mu \mathrm{m}$ again bridged the two electrodes. The mechanical signals are produced by light human finger taps. Two taps are made at each location along the length of the film, before moving on the next position. Initiation of the tap is indicated by a spike in current. When perturbations are made on the left electrode, a positive change in current is seen. It is observed that there is a gradual change in direction and amplitude of the current generated with perturbations initiating exactly on the working electrode, then along the length of the hydrogel to the end with the counter electrode. The response changes from being positive on the working electrode to negative on the counter electrode with varying directions in the region between them. The amplitude diminishes as the perturbations move away from either of the electrodes and is the smaller for perturbations in the middle of the sensor strip. Note the sharp and nearly instantaneous change in current. For example for perturbations on the left hand side the current is first downwards then upwards, all 
within $20 \sim 30 \mathrm{~ms}$, and the reverse is seen for perturbations on the right had side, similar to the estimated time of contact. The polarity of the current response is consistent with PF6- ions being more mobile than $\mathrm{Na}^{+}$.

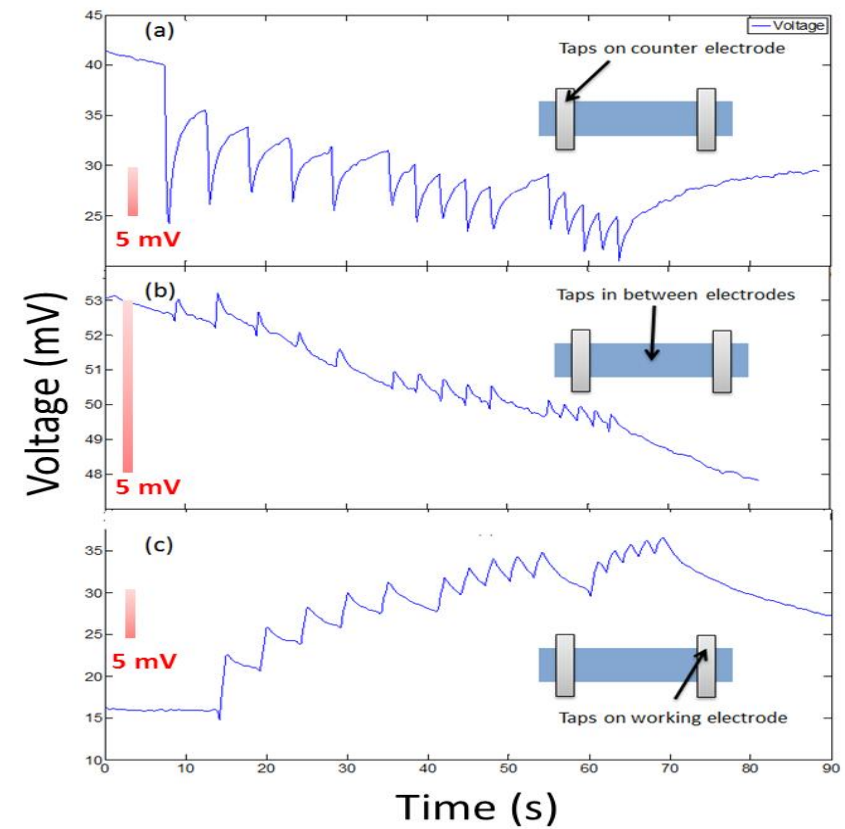

Figure 4: Open circuit potential response for $\mathrm{KCl} 1.0 \mathrm{M}$ electrolyte and taps on (a) counter electrode, (b) between electrodes and (c) on the working electrode

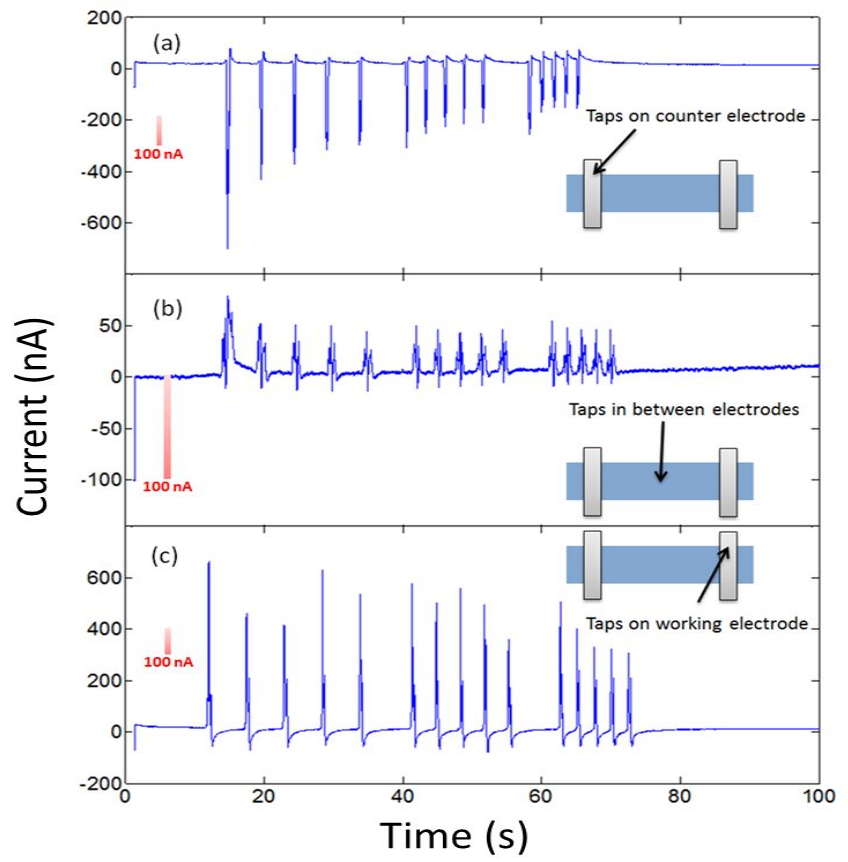

Figure 5: Short circuit current response for $\mathrm{KCl} 1.0 \mathrm{M}$ electrolyte and taps on (a) counter electrode, (b) between electrodes and (c) on the working electrode 


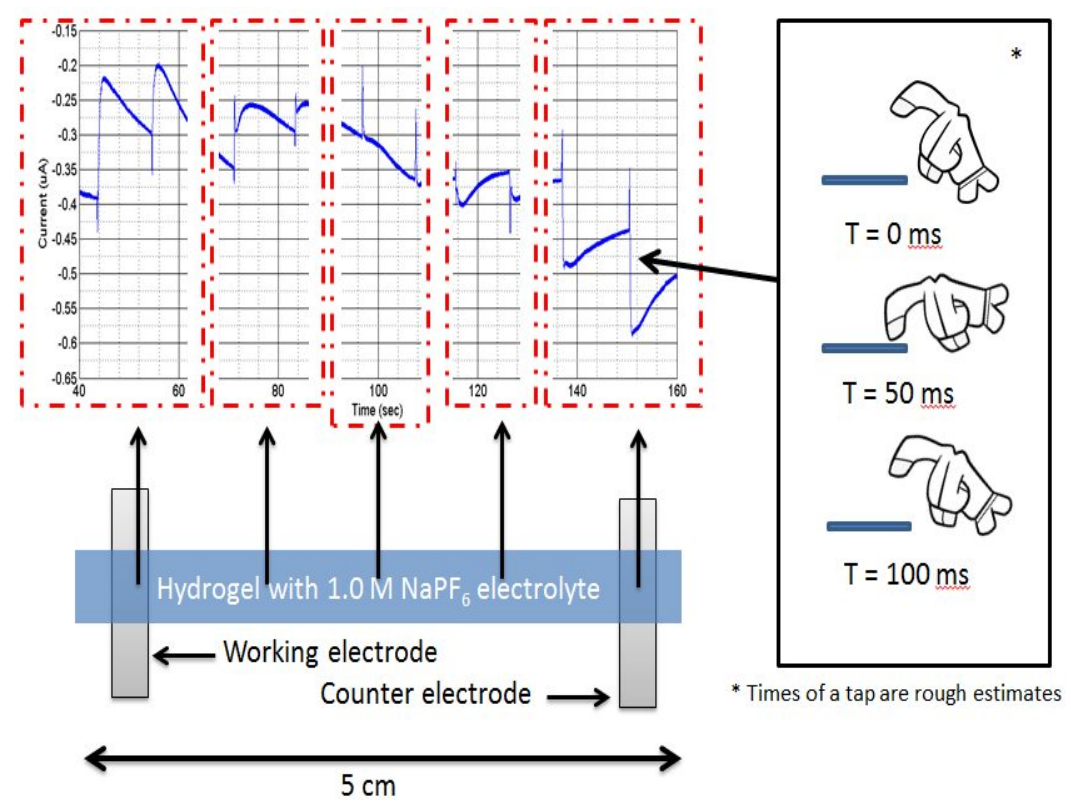

Figure 6: Response of hydrogel sensor to finger taps along its length

\section{DISCUSSION}

Four key characteristics of the response observed in the results sections are now discussed: (1) The speed of voltage and current response; (2) The amplitude of subsequent voltage/current responses perturbations with perturbations frequencies (3) The direction of voltage and current generated; (4) The amplitude of the voltage and current generated on electrodes relative to the locations of perturbations in between electrodes.

\section{Speed of voltage and current response}

It has been observed that the hydrogel sensor responds almost instantaneously to a mechanical stimulus and the signal rise time is as small as $25 \mathrm{~ms}$ followed by a slower process of returning to steady state as seen in Figure 4 and Figure 5. Possible factors responsible for this may be a slow form of a mechanical relaxation process of the hydrogel sensor body combined with the diffusion coefficient of ions in the electrolyte.

\section{Amplitude of subsequent perturbations with perturbation frequencies}

It is observed from experimental data that in case of faster perturbations, the amplitude of the response decays. A possible cause of this is mechanical relaxation time of the gel as well as the hysteresis properties of the gel. To recover the original amplitude of response it appears that the sensor requires returning to the steady undistorted state with the ions in equilibrium in terms of charge and concentration. In comparison, if the hydrogel has not fully recovered original state and there is already an existing deformation, the further deformation and charge separation caused under these conditions is less effective, hence a smaller amplitude of response. There is also the possibility of exudation of the electrolyte with a perturbation and then the subsequent reabsorption which may be responsible for the slower return to equilibrium, or in general a flux of electrolyte within the gel. The sensor material has a Young's modulus of a few hundred $\mathrm{kPa}$ and the pressure applied is roughly $30 \mathrm{kPa} \sim 50 \mathrm{kPa}$ which deforms the sensor by roughly $30 \%$, so stiffer materials or lighter touches may recover faster and or show less drift.

\section{Polarity of voltage generated}

The observed polarity further supports the ion displacement hypothesis presented here to explain the working principle of the sensor. It is observed in case of $\mathrm{KCl}$ as an electrolyte, from Figure 4 (a) that perturbations on the counter electrode 
causes the voltage to go down i.e. the counter electrode becomes relatively less positive with reference to the working electrode, while in Figure 4 (c) the voltage goes up with perturbations on the working electrode. This validates the claim that the generation of the voltage is due to the physical displacement of ions bringing about a difference in charge concentration at the location of the perturbation compared to a reference location away from the perturbation. It is observed that in case of $\mathrm{KCl}$ the direction of current was the direction of propagation of more mobile ion (the positive ion $\mathrm{K}^{+}$) while in case of NaPF6 it was the negative ion i.e. PF6. In NaPF6 the solvation sphere of the $\mathrm{Na}^{+}$ion causes it to be less mobile than expected and hence the resulting direction of current. Processes in addition to diffusion may be involved. For example it is possible that there is convection of solvent within the gel on contact, or that differential ion mobility leads some ions to move faster than others on the application of pressure.

\section{Amplitude of voltage generated on electrode compared to away from the electrodes}

It is easily observable from Figure 6 that when the perturbations occur on or near the readout electrodes the amplitude is larger in comparison to perturbations away from the readout electrodes. In fact, away from the electrodes it might be expected that ions would be displaced equally in both directions creating no net voltage or current. Instead, some signal is observed, albeit attenuated. This is perhaps due to pressure waves emanating from the contact point. Simple passive readout circuits have been implemented for the readout process - a charge amplifier for example - avoiding the use of expensive electronics and the need for applying power to the device itself.

While our materials are quite tough, others have demonstrated even more robust and transparent hydrogels that are appropriate for sensing application. These can be stretched to 21 times their original length before failure [29] in one example. Transparency in water of $98.9 \%$ has been observed [30] in similar $11 \mathrm{~mm}$ thick polyacrylamide based hydrogel. The gels need to remain wet which necessitates encapsulation that is as flexible as the gel itself. Possibilities are being explored including PDMS, poly(styrene-b-isobutylene-b-styrene) block copolymer (SIBS) [31], and parylene. Tuning and optimization of the mechanical and ion transport properties as well as demonstration of encapsulation are important next steps in the effort to make useful and high performance sensors.

\section{CONCLUSION}

We have demonstrated a proof-of-principle piezoionically driven touch sensor based on polyurethane hydrogel swollen with various salt solutions. The hydrogel reliably produced voltage and current signals in response to differential internal pressure induced by local compression. The open circuit potential and short circuit current measured were on the order of $10^{-2} \mathrm{~V}$ and $10^{-7} \mathrm{~A}$. Voltages are lower but currents are higher than are expected of piezoelectric materials of similar dimensions such as PVDF. It is also demonstrated that the signal response is characteristic of the location at which the deformation is applied. On the device level, the compliant, transparent and low cost nature of the sensor materials may make them attractive for use as distributed sensors in the home, integration into soft robots use in wearable sensors and perhaps even stretchable interactive interfaces for electronic devices.

\section{ACKNOWLEDGEMENT}

This work was supported in part by the Institute for Computing, Information and Cognitive Systems (ICICS) at UBC.

\section{REFERENCES}

[1] C. Liu, Foundations of MEMS. Pearson Prentice Hall, 2006, p. 530.

[2] D. J. Beebe, A. S. Hsieh, D. D. Denton, and R. G. Radwin, "A silicon force sensor for robotics and medicine," Sensors Actuators A Phys., vol. 50, no. 1-2, pp. 55-65, Aug. 1995.

[3] M. R. Wolffenbuttel and P. P. L. Regtien, "Polysilicon bridges for the realization of tactile sensors," Sensors Actuators A Phys., vol. 26, no. 1-3, pp. 257-264, Mar. 1991.

[4] S. Tadigadapa and K. Mateti, "Piezoelectric MEMS sensors: state-of-the-art and perspectives," Meas. Sci. Technol., vol. 20, no. 9, p. 092001, Sep. 2009.

[5] J. -i. Yuji and C. Sonoda, "A PVDF Tactile Sensor for Static Contact Force and Contact Temperature," in 2006 5th IEEE Conference on Sensors, 2006, pp. 738-741. 
[6] W. P. Eaton and J. H. Smith, "Micromachined pressure sensors: review and recent developments," in Smart Structures and Materials '97, 1997, pp. 30-41.

[7] L. K. Baxter, Capacitive Sensors: Design and Applications. John Wiley \& Sons, 1996, p. 320.

[8] P. A. Schmidt, E. Maël, and R. P. Würtz, "A sensor for dynamic tactile information with applications in humanrobot interaction and object exploration," Rob. Auton. Syst., vol. 54, no. 12, pp. 1005-1014, Dec. 2006.

[9] J.-S. Heo, J.-H. Chung, and J.-J. Lee, "Tactile sensor arrays using fiber Bragg grating sensors," Sensors Actuators A Phys., vol. 126, no. 2, pp. 312-327, Feb. 2006.

[10] E. Torres-Jara, I. Vasilescu, and R. Coral, "A soft touch: Compliant Tactile Sensors for Sensitive Manipulation," Mar. 2006.

[11] S. C. B. Mannsfeld, B. C.-K. Tee, R. M. Stoltenberg, C. V. H.-H. Chen, S. Barman, B. V. O. Muir, A. N. Sokolov, C. Reese, and Z. Bao, "Highly sensitive flexible pressure sensors with microstructured rubber dielectric layers.," Nat. Mater., vol. 9, no. 10, pp. 859-64, Oct. 2010.

[12] D. J. Lipomi, M. Vosgueritchian, B. C.-K. Tee, S. L. Hellstrom, J. A. Lee, C. H. Fox, and Z. Bao, "Skin-like pressure and strain sensors based on transparent elastic films of carbon nanotubes.," Nat. Nanotechnol., vol. 6, no. 12 , pp. 788-92, Dec. 2011.

[13] W. Hu, X. Niu, R. Zhao, and Q. Pei, "Elastomeric transparent capacitive sensors based on an interpenetrating composite of silver nanowires and polyurethane," Appl. Phys. Lett., vol. 102, no. 8, p. 083303, Feb. 2013.

[14] J.-Y. Sun, C. Keplinger, G. M. Whitesides, and Z. Suo, "Ionic skin," Adv. Mater., Oct. 2014.

[15] D. Beebe, J. Moore, J. Bauer, Q. Yu, R. Liu, C. Devadoss, and B. Jo, "Functional hydrogel structures for autonomous flow control inside microfluidic channels," Nature, vol. 404, no. 6778, pp. 588-90, Apr. 2000.

[16] K.-F. Arndt, D. Kuckling, and A. Richter, "Application of sensitive hydrogels in flow control," Polym. Adv. Technol., vol. 11, no. 8-12, pp. 496-505, Aug. 2000.

[17] H. Tokuyama and Y. Kato, "Preparation of poly(N-isopropylacrylamide) emulsion gels and their drug release behaviors.," Colloids Surf. B. Biointerfaces, vol. 67, no. 1, pp. 92-8, Nov. 2008.

[18] L. Wang, J. Zhang, and A. Wang, "Removal of methylene blue from aqueous solution using chitosan-gpoly(acrylic acid)/montmorillonite superadsorbent nanocomposite," Colloids Surfaces A Physicochem. Eng. Asp., vol. 322, no. 1-3, pp. 47-53, Jun. 2008.

[19] L. Dong, A. K. Agarwal, D. J. Beebe, and H. Jiang, "Adaptive liquid microlenses activated by stimuli-responsive hydrogels.," Nature, vol. 442, no. 7102, pp. 551-4, Aug. 2006.

[20] J. P. Gong, "Friction and lubrication of hydrogels?its richness and complexity," Soft Matter, vol. 2, no. 7, p. 544, Jun. 2006.

[21] N. Hirota, Y. Kumaki, T. Narita, J. P. Gong, and Y. Osada, "Effect of Charge on Protein Diffusion in Hydrogels," J. Phys. Chem. B, vol. 104, no. 42, pp. 9898-9903, Oct. 2000.

[22] W. Takashima, T. Uesugi, M. Fukui, M. Kaneko, and K. Kaneto, "Mechanochemoelectrical effect of polyaniline film," Synth. Met., vol. 85, no. 1-3, pp. 1395-1396, Mar. 1997.

[23] Y. Wu, G. Alici, J. D. W. Madden, G. M. Spinks, and G. G. Wallace, "Soft mechanical sensors through reverse actuation in polypyrrole," Adv. Funct. Mater., vol. 17, no. 16, pp. 3216-3222, 2007.

[24] G. Alici, G. M. Spinks, J. D. Madden, Y. Wu, and G. G. Wallace, "Response Characterization of Electroactive Polymers as Mechanical Sensors," IEEE/ASME Trans. Mechatronics, vol. 13, no. 2, pp. 187-196, Apr. 2008.

[25] T. Shoa, J. D. W. Madden, T. Mirfakhrai, G. Alici, G. M. Spinks, and G. G. Wallace, "Electromechanical coupling in polypyrrole sensors and actuators," Sensors Actuators A Phys., vol. 161, no. 1-2, pp. 127-133, Jun. 2010.

[26] M. Shahinpoor, "New effect in ionic polymeric gels: the ionic flexogelectric effect," in Smart Structures \& Materials '95, 1995, pp. 42-53.

[27] B. Bhandari, G.-Y. Lee, and S.-H. Ahn, "A review on IPMC material as actuators and sensors: Fabrications, characteristics and applications," Int. J. Precis. Eng. Manuf., vol. 13, no. 1, pp. 141-163, Feb. 2012.

[28] M. Otsuki, T. Okuyama, and M. Tanaka, "Characteristic evaluation of a solid polymer electrolyte sensor," Microsyst. Technol., vol. 17, no. 5-7, pp. 1129-1133, Apr. 2011.

[29] J.-Y. Sun, X. Zhao, W. R. K. Illeperuma, O. Chaudhuri, K. H. Oh, D. J. Mooney, J. J. Vlassak, and Z. Suo, "Highly stretchable and tough hydrogels.," Nature, vol. 489, no. 7414, pp. 133-6, Sep. 2012.

[30] C. Keplinger, J.-Y. Sun, C. C. Foo, P. Rothemund, G. M. Whitesides, and Z. Suo, "Stretchable, transparent, ionic conductors.," Science, vol. 341, no. 6149, pp. 984-7, Aug. 2013.

[31] S. Naficy, N. Stoboi, P. G. Whitten, G. M. Spinks, and G. G. Wallace, "Evaluation of encapsulating coatings on the performance of polypyrrole actuators," Smart Mater. Struct., vol. 22, no. 7, p. 075005, Jul. 2013. 